\title{
A Mobile App to Improve Patient Management in Emergency Departments: Caregiver Needs Analysis, Design and Early Technology Acceptance Assessment
}

\author{
Frédéric EHRLER ${ }^{\mathrm{a}}$, Carlotta TUOR ${ }^{\mathrm{b}}$, Robin REY ${ }^{\mathrm{b}}$, and Johan N. SIEBERT ${ }^{\mathrm{c}, 1}$ \\ ${ }^{a}$ Division of Medical Information Sciences, Geneva University Hospitals, \\ Geneva, Switzerland \\ ${ }^{\mathrm{b}}$ University of Geneva, Faculty of Medicine, Geneva, Switzerland \\ ${ }^{\mathrm{c}}$ Department of Pediatric Emergency Medicine, Children's Hospital, Geneva \\ University Hospitals, Geneva, Switzerland
}

\begin{abstract}
Emergency care is very complex in that it requires patient-centered care in a coordinated manner among multiple providers in a highly distractible, unpredictable and stressful environment. Sharing information efficiently between providers in this context is difficult. Connecting emergency providers with each other through a digital communication channel could improve the efficiency of information sharing and emergency care. This study describes the development process of PIMPmyHospital, a mobile app dedicated to emergency department physicians and nurses to collaboratively manage their patients. We relied on a usercentered design process involving caregivers from a pediatric emergency department. The process started with semi-structured interviews that informed the specifications of the app, followed by an iterative design and development approach. The resulting prototype was evaluated by end-users using the perceived usefulness dimension of the technology acceptance model questionnaire. Early user engagement during the design and development of a dedicated mobile app must be taken into account to improve its perceived usefulness and future adoption.
\end{abstract}

Keywords. Emergency Medicine, Information Technology, Interprofessional Relations, Mobile Applications, Patient Care Management

\section{Introduction}

An emergency department (ED) is a place that requires high functional operability. This involves dealing with an erratic patient flow, including life-threatening emergencies requiring immediate attention, while simultaneously providing safe and optimal care to all patients in a timely manner in an endemically overcrowded and resource-constrained environment. In this context, time wasted by caregivers on indirect patient and nonpatient activities wastes resources to the detriment of patient workflow, staff satisfaction and, ultimately, patients. It has been observed that ED physicians and nurses spend nearly

\footnotetext{
${ }^{1}$ Corresponding Author, Johan N. Siebert, Department of Pediatric Emergency Medicine, Children's Hospital, Geneva University Hospitals, 47 Avenue de la Roseraie, 1205 Geneva, Switzerland; e-mail: Johan.Siebert@hcuge.ch
} 
half of their time on indirect patient and non-patient activities, including traveling within the unit, getting test results, and locating colleagues to share medical information $[1 ; 2]$. It is also challenging for ED caregivers to maintain a high level of awareness of each patient's individual situation while multitasking in a highly distracting and rapidly changing environment. For example, clinicians may not be notified instantly that a perfusion has ended or nurses that laboratory tests were prescribed. As a result, obtaining real-time patient information and sharing it between caregivers can be suboptimal in terms of communication, efficiency and patient flow. Patients have to wait unnecessarily in the emergency room when they could have been sent home or hospitalized earlier, which would improve ED patient turnover and potentially reduce upstream waiting times. Evidence to demonstrate the benefits of computerized tools specifically dedicated to streamlining shared patient management to overcome these drawbacks in ED is scarce.

To address this issue, we developed a mobile application (app) dedicated to ED caregivers, PIMPmyHospital (Patients In My Pocket in my Hospital). This app aims to provide relevant information in real-time about the patients they care for, as well as a secure chat and messaging platform to virtually connect physicians and nurses caring for the same patients. We present here the caregivers' needs analysis, design and early technology acceptance of this app by ED physicians and nurses.

\section{Methods}

The design and development of the app entailed a user-centered and multidisciplinary design approach. A four-step development cycle was used.

\subsection{Semi-structured interview}

In the first step, interviews were conducted with pediatric ED physicians and nurses of a tertiary hospital with more than 33,000 visits per year. After obtaining consent from each participant, individual semi-structured, face-to-face interviews with 20 open-ended questions were carried out to explore ED caregivers' perceptions of everyday challenges. Interviews were conducted by two qualified investigators (CT and RR) that were not involved in the aspects of emergency care with the participants. All questions were asked to participants in the same order and each participant was blinded to all other participants' answers. Interviews were audio-recorded, anonymized, and transcribed verbatim. An in-depth thematic analysis was then conducted according to the six-step iterative framework [3, 4] to identify patterns of emerging themes, including: 1) familiarization with data by reading the transcripts; 2) assigning preliminary codes to interesting features of the data in a systematic fashion across the entire data set; 3) searching for themes among the codes across the interviews; 4) reviewing themes by organizing the data that can best fit together into sub-themes; 5) defining and naming final themes; and 6) producing the report. The first five steps were blinded between the two interviewers. Findings were reported according to the Consolidated Criteria for Reporting Qualitative Research recommendations (available upon request) [5].

\subsection{App's functional specifications identification}

The app's specifications were defined based on the thematic analysis described above. Iterative focus group sessions involving three computer scientists and two pediatric 
emergency physicians, including those from the project team, were conducted to analyze the feasibility of transcribing these specifications into functionality in the core app.

\subsection{Design and prototyping}

Relevant specifications gathered from the previous stages informed the design and development of the app prototype. Static mock-ups were initially produced with Adobe $\mathrm{XD}$ (Adobe Inc) to visualize the information displayed in a format similar to that of the proposed mobile app. The design sessions were an iterative process in which the project team provided feedback until the prototype was considered as covering all functions identified as relevant.

\subsection{Evaluation}

In the final step, we conducted a preliminary evaluation of the prototype through interviews using the Technology Acceptance Model (TAM) as a guiding theoretical framework to provide insight into one key determinant that influences user acceptance of technology, i.e., perceived usefulness [6]. Seven-point Likert-type scales ranging from "strongly disagree" (1) to "strongly agree" (7) were used to assess the six statements related to participants' perceived usefulness of the app. We reported average scores on each statement and the internal consistency of the questionnaire was measured using Cronbach's coefficient alpha. Finally, participants were asked to provide oral feedback on the app, which was transcribed to identify expected benefits and potential drawbacks.

\subsection{Ethical considerations}

As this study was considered as falling outside of the scope of Swiss legislation regulating research on human subjects, the need for local ethics committee approval was waived (No. req-2021-00740).

\section{Results}

\subsection{Interviews}

Between June 8 and June 29, 2021, a convenience sample of participants was recruited and interviewed. Data saturation [7] was reached by 14 interviews. However, interviews were continued until 20 participants to ensure a maximum variation in interviewees. This included eight ED fellows, two postgraduate year-1 residents, and 10 ED nurses. Mean (standard deviation) age, years since certification, and years spent in the ED were 37.0 (8.1), 12.0 (8.8), and 6.0 (5.4), respectively. Interviews were completed by all participants without any missing items (average duration, 19.6 [range 9.6-31.7] minutes per participant [total 392 minutes]). Thematic analysis of the transcripts enabled the identification of four main themes (Table 1) as follows:

Theme 1: Mobility. Caregivers reported that they have to travel extensively. Places that bring caregivers together seemed conducive to fostering communication. 
Design principle. Reducing back and forth flow would 1) streamline caregiver's activities, 2) avoid staff stress and fatigue, and 3) reduce discontinuity of care. The app was tailored to caregivers by connecting them virtually and providing them with targeted information in real time. In other words, the app was designed so that information reaches the caregiver rather than the caregiver having to reach out for the information.

Theme 2: Time optimization. Almost all participants (19/20;95\%), irrespective of their functions as physicians or nurses, noted the need to reduce the waste of time spent to find the other caregivers involved in the care of the patients. They also pointed out the need to quickly know who is caring for a specific patient.

Design principle. The design principle for time optimization was to 1) focus on patients managed by a given caregiver, 2) facilitate the identification of other caregivers caring for the same patients, and 3) provide the ability to reach them quickly. To address these needs, we have integrated a patient list tailored to each caregiver. Only the patients the caregiver is in charge of are displayed in real time on the app, with other caregivers sharing the same patients displayed to the right, together with the time since ED admission (Figure 1). The entry and exit of patients on the list are automatically controlled by the institution's patient management software linked to the app, thus requiring no user intervention. Other caregivers in charge of the same patient are represented by their initials in a color-coded box indicating their functions (i.e., physician/red, nurse/blue, etc.). A click on the box opens an instant chat system to reach them remotely.
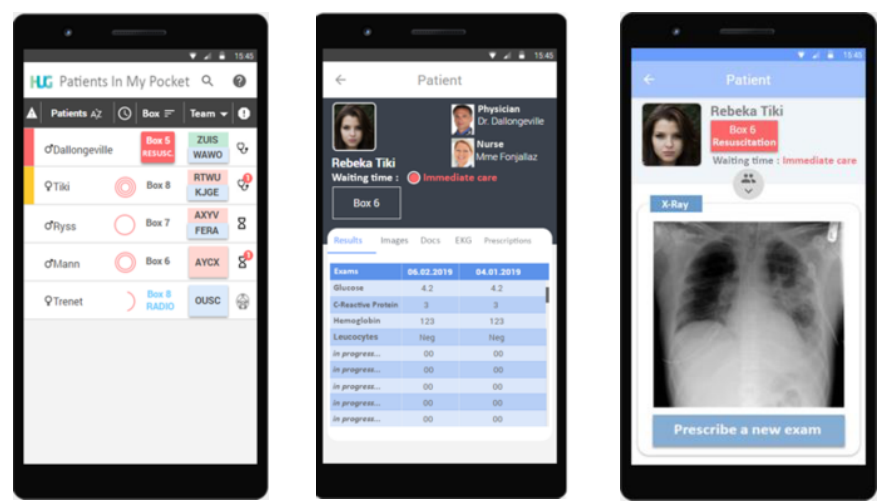

Figure 1. Prototype of the PIMPmyHospital app. Left screenshot: color-coded bars represent the five-level Canadian Triage and Acuity Scale [8]); patient gender and identity; time since admission as radial circular timers; patient allocation per room; color-coded box of individuals in charge of the patient; patient status (seen by a physician, waiting for results, CT-scan in progress, etc.). Push notifications prompt the user to be aware of the situations. Middle and right screenshots: selecting a patient opens a new page with scrollable contextual tab menus containing information related to laboratory results, imaging, patient files, electrocardiograms, and prescriptions entered in the computerized institutional prescription software.

Theme 3: Medical information. Almost all participants agreed that it was more convenient to access medical information on-the-go via their mobiles compared to static computers at disposal. The consequences of missing or delayed laboratory test results and, to a lesser extent, radiological results, was a common concern as no alert is generated directly to the caregiver. This was considered by many as a hindrance to patient 
flow and a source of distraction and cognitive overload as they had to remember to check the results several times until they were made available.

Design principle. Simple, real-time display of laboratory and radiology results with prompts that integrate into the workflow can 1) help caregivers focus on their tasks while waiting for their availability, and 2) access them in a timely manner, wherever they are. To achieve this goal, we made these results available to caregivers by synchronizing and displaying them directly on the app, with prompts (Figure 1).

Theme 4: Communication. The caregivers recognized the practicality of the electronic ED patient status board already in place in the ED as a core medium for communicating via text messages and providing situational awareness. However, they also pointed out constraints linked to this multi-channel and disparate display of all ED patients at a glance, making reading complicated and at risk of fragmented or omitted information. They noted both the lack of push notifications to inform caregivers of incoming messages and validation of their receipt. Caregivers also expressed their desire to be able to communicate directly with others in charge of their patients, thus justifying the need for a complementary medium.

Design principle. Enabling caregivers to use a single and responsive communication channel could make care more efficient by promoting instantaneous and shared information exchange, while reducing fragmentation and information loss. To address this design principle, we created a chat area (Figure 2). To ensure responsiveness, push notifications were designed to inform caregivers of incoming messages.
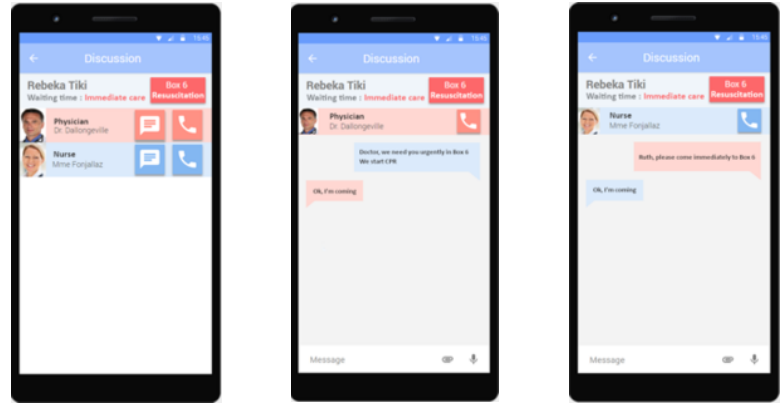

Figure 2. Prototype of the instant messaging system. The logos at the bottom of the screen represent the possibility to also import and link documents to the conversion, as well as to send voice memos.

\subsection{Preliminary evaluation}

The reliability of the questionnaire was excellent with a Cronbach's alpha of 0.98 for perceived usefulness. The scores for perceived usefulness suggested that the participants strongly agreed that the app is a useful tool (Table 1). Based on user feedback, the shared aspect of the information assisted by a mobile app was seen by many as beneficial in facilitating communication in a coordinated and collaborative manner. Although participants were likely to use the app, they also anticipated some potential drawbacks, such as being overwhelmed by messages occurring at inopportune times or that communication via the app would come at the expense of oral transmission. 
Table 1. Items and scores for the perceived usefulness of the mobile app PIMPmyHospital

\begin{tabular}{lcc}
\hline Perceived usefulness & Mean & $\begin{array}{c}\text { Standard } \\
\text { Deviation }\end{array}$ \\
\hline $\begin{array}{l}\text { 1. Using this app in my job would enable me to accomplish } \\
\text { tasks more quickly }\end{array}$ & 5.75 & 1.02 \\
2. Using this app would improve the quality of & 5.50 & 1.10 \\
my work in providing better patient care & & \\
3. Using this app in my job would increase my productivity & 5.40 & 1.57 \\
4. Using this app would enhance my effectiveness on the job & 5.45 & 1.50 \\
5. Using this app would make my job easier to perform & 6.0 & 1.26 \\
6. I would find this app useful in my job & 5.7 & 1.46 \\
Overall & $\mathbf{5 . 6 3}$ & $\mathbf{1 . 3 2}$ \\
\hline
\end{tabular}

\section{Discussion}

EDs are highly dynamic and stressful care environments subject to overcrowding and acute situations requiring rapid and coordinated care among several stakeholders. In this context, wasted time, discontinuity of care, suboptimal communication, and omission or delay in seeking information can compromise patient outcomes and safety. This study sought to develop a user-centered mobile app connecting ED caregivers to each other. Preliminary results are encouraging, with users rating the usefulness of the app as high for a newly-developed digital tool. This outcome should however be validated through a formal evaluation in simulated setting before being used in real-life situations.

\section{Conclusion}

Early user engagement during the design and development of a dedicated mobile app is a crucial step to improve its perceived usefulness and future adoption. Providing ED caregivers with a communication and information sharing mobile app can be a light and affordable strategy to promote ED efficiency and improve patient care.

\section{References}

[1] Hollingsworth JC, Chisholm CD, Giles BK, Cordell WH, Nelson DR. How do physicians and nurses spend their time in the emergency department?, Ann Emerg Med 31 (1998), 87-91.

[2] Weigl M, Handl T, Wehler M, Schneider A. Time-allocation study of nurse and physician activities in the emergency department. Med Klin Intensivmed Notfmed 116 (2021), 229-237.

[3] Kiger ME, Varpio L. Thematic analysis of qualitative data: AMEE Guide No. 131, Med Teach 42 (2020), 846-854.

[4] Maguire M, Delahunt B. Doing a thematic analysis: A practical, step-by-step guide for learning and teaching scholars, All Ireland Journal of Higher Education 9 (2017).

[5] Tong A, Sainsbury P, Craig J. Consolidated criteria for reporting qualitative research (COREQ): a 32item checklist for interviews and focus groups, Int J Qual Health Care 19 (2007), 349-357.

[6] Davis FD. Perceived Usefulness, Perceived Ease of Use, and User Acceptance of Information Technology, MIS Quarterly 13 (1989), 319-340.

[7] Saunders B, Sim J, Kingstone T, Baker S, Waterfield J, Bartlam B, Burroughs H, Jinks C. Saturation in qualitative research: exploring its conceptualization and operationalization, Qual Quant 52 (2018), 18931907.

[8] Beveridge R. CAEP issues. The Canadian Triage and Acuity Scale: a new and critical element in health care reform. Canadian Association of Emergency Physicians, J Emerg Med 16 (1998), 507-511. 